\title{
Localization length and impurity dielectric susceptibility in the critical regime of the metal-insulator transition in homogeneously doped $p$-type Ge
}

\author{
Michio Watanabe, ${ }^{1}$ Kohei M. Itoh, ${ }^{1,2}$ Youiti Ootuka ${ }^{3}$, and Eugene E. Haller ${ }^{4}$ \\ ${ }^{1}$ Department of Applied Physics and Physico-Informatics, Keio University, Yokohama 223-8522, Japan \\ ${ }^{2}$ PRESTO-JST, Yokohama 223-8522, Japan \\ ${ }^{3}$ Institute of Physics, University of Tsukuba, 1-1-1 Tennodai, Tsukuba, Ibaraki 305-8571, Japan \\ ${ }^{4}$ Lawrence Berkeley National Laboratory and University of California at Berkeley, Berkeley, California 94720
}

(Received 10 April 2000)

\begin{abstract}
We have determined the localization length $\xi$ and the impurity dielectric susceptibility $\chi_{\text {imp }}$ as a function of $\mathrm{Ga}$ acceptor concentrations $(N)$ in nominally uncompensated ${ }^{70} \mathrm{Ge}$ : Ga just below the critical concentration $\left(N_{c}\right)$ for the metal-insulator transition. Both $\xi$ and $\chi_{\text {imp }}$ diverge at $N_{c}$ according to the functions $\xi \propto\left(1-N / N_{c}\right)^{-\nu}$ and $\chi_{\mathrm{imp}} \propto\left(N_{c} / N-1\right)^{-\zeta}$, respectively, with $\nu=$ $1.2 \pm 0.3$ and $\zeta=2.3 \pm 0.6$ for $0.99 N_{c}<N<N_{c}$. Outside of this region $\left(N<0.99 N_{c}\right)$, the values of the exponents drop to $\nu=0.33 \pm 0.03$ and $\zeta=0.62 \pm 0.05$. The effect of the small amount of compensating dopants that are present in our nominally uncompensated samples, may be responsible for the change of the critical exponents at $N \approx 0.99 N_{c}$.
\end{abstract}

PACS numbers: $71.30 .+\mathrm{h}, 72.80 . \mathrm{Cw}$

The metal-insulator transition (MIT) in doped semiconductors is a unique quantum phase transition in the sense that both disorder and electron-electron interaction play a key role. 1 Important information about the MIT is provided by the values of the critical exponents for the zero-temperature conductivity, correlation length, localization length, and impurity dielectric susceptibility. From a theoretical point of view, the correlation length in the metallic phase and the localization length in the insulating phase diverge at the critical point with the same exponent $\nu$, i.e., they are proportional to $\left|N / N_{c}-1\right|^{-\nu}$ in the critical regime of the MIT. ( $N$ is the dopant concentration and $N_{c}$ is the critical concentration for the MIT.) Since direct experimental determination of $\nu$ is extremely difficult, researchers have usually determined, instead of $\nu$, the value of $\mu$ defined by $\sigma(0) \propto\left(N / N_{c}-1\right)^{\mu}$ where $\sigma(0)$ is the conductivity extrapolated to $T=0.2 \mathrm{It}$ is also possible to evaluate $\mu$ from finite-temperature scaling of the form $\sigma(N, T) T^{\propto} T^{x} f\left(\left|N / N_{c}-1\right| / T^{y}\right)$ where $x / y$ is equivalent to $\mu$. 6 Values of $\nu$ are then obtained assuming $\nu=\mu$ for three-dimensional systems.

In this work we have determined directly the localization length $\xi$ and the impurity dielectric susceptibility $\chi_{\mathrm{imp}}$ in neutron-transmutation-doped (NTD), nominally uncompensated ${ }^{70} \mathrm{Ge}$ :Ga just below $N_{c}$. The application of NTD to isotopically enriched ${ }^{70} \mathrm{Ge}$ leads to unsurpassed doping homogeneity and precisely controlled doping concentration. As a result, we have been able to approach the transition as close as $0.999 N_{c}$ from the insulating side and $1.0004 N_{c}$ from the metallic side. In zero magnetic field, the low-temperature resistivity of the samples is described by variable-range hopping (VRH) conduction within the Coulomb gap. field and temperature dependence of the resistivity are subsequently measured in order to determine directly $\xi$ and $\chi_{\text {imp }}$ in the context of the VRH theory. 8

This kind of determination of $\xi$ and $\chi_{\text {imp }}$ was per- formed for compensated Ge:As by Ionov et al. $\mathrm{g}^{\mathrm{S}}$ They found $\xi \propto\left(1-N / N_{c}\right)^{-\nu}$ and $\chi_{\mathrm{imp}} \propto\left(N_{c} / N-1\right)^{-\zeta}$ with $\nu=0.60 \pm 0.04$ and $\zeta=1.38 \pm 0.07$, respectively, for $N<0.96 N_{c}$. The significance of their result is the experimental verification of the relation $2 \nu \approx \zeta$ that had been predicted by scaling theories.10 11 However, the critical exponents of compensated samples are known to be different from those of nominally uncompensated samples. Therefore, the present work which probes $\xi$ and $\chi_{\text {imp }}$ in nominally uncompensated samples is relevant for the fundamental understanding of the MIT. The previous effort to measure $\chi_{\mathrm{imp}}$ has also contributed.12 14 Hess et al. found $\zeta=1.15 \pm 0.15$ in nominally uncompensated Si:P.13 Since $\mu \approx 0.5$ was determined for the same series of Si:P samples, $2 \mu \approx \zeta$ was again valid. Katsumoto has found $\zeta \approx 2$ and $\mu \approx 1$ for compensated $\mathrm{Al}_{x} \mathrm{Ga}_{1-x} \mathrm{As}: \mathrm{Si}$, i.e., again, $2 \mu \approx \zeta$ applies. 14 Thus, in these cases the conclusion $2 \nu \approx \zeta$ was reached indirectly, by assuming $\mu=\nu$. The work reported here, on the other hand, determines $\nu$ directly, i.e., we do not have to rely on the assumption $\mu=\nu$ in order to study the behavior of $\xi$ near $N_{c}$.

All of the ${ }^{70} \mathrm{Ge}$ :Ga samples used in this study were prepared by NTD of isotopically enriched ${ }^{70} \mathrm{Ge}$ single crystals. We use the NTD process since it is knoyn to produce the most homogeneous dopant distribution. Details of the sample preparation and characterization are described elsewhere. 3 In this study, we determined the low-temperature $(0.05-0.5 \mathrm{~K})$ resistivity of nine samples in weak magnetic fields $(<0.4 \mathrm{~T})$ applied in the direction perpendicular to the current flow.

The electrical conduction of doped semiconductors on the insulating side of the MIT is often dominated by VRH at low temperatures. The temperature dependence of the resistivity $\rho(T)$ for VRH is written in the form of

$$
\rho(T)=\rho_{0}(T) \exp \left[\left(T_{0} / T\right)^{p}\right]
$$

where $p=1 / 2$ for the excitation within a parabolic- 
shaped energy gap (the Coulomb gap), 8 and $p=14$ for a constant density of states around the Fermi level.15 In our earlier work, 3 we reported that $p=1 / 2$ for $N<0.991 N_{c}$ $\left(N_{c}=1.860 \times 10^{17} \mathrm{~cm}^{-3}\right)$ and that $p$ decreases rapidly as $N$ approaches $N_{c}$ from $0.991 N_{c}$ and becomes even smaller than $1 / 4$ when we neglect the temperature variation of $\rho_{0}(T)$. However, the variation contributes greatly to the temperature dependence of $\rho(T)$ near $N_{c}$ because the factor $T_{0} / T$ in the exponential terms become very small, i.e., the temperature dependencies of $\rho_{0}(T)$ and that of the exponential term become comparable. Theoretically, $\rho_{0}(T)$ is expected to vary as $\rho_{0} \propto T^{-r}$ but the value of $r$ including the sign has not been derived yet for $\mathrm{VRH}$ with both $p=1 / 2$ (Ref. 16) and $p=1 / 4$ (Ref. 17).
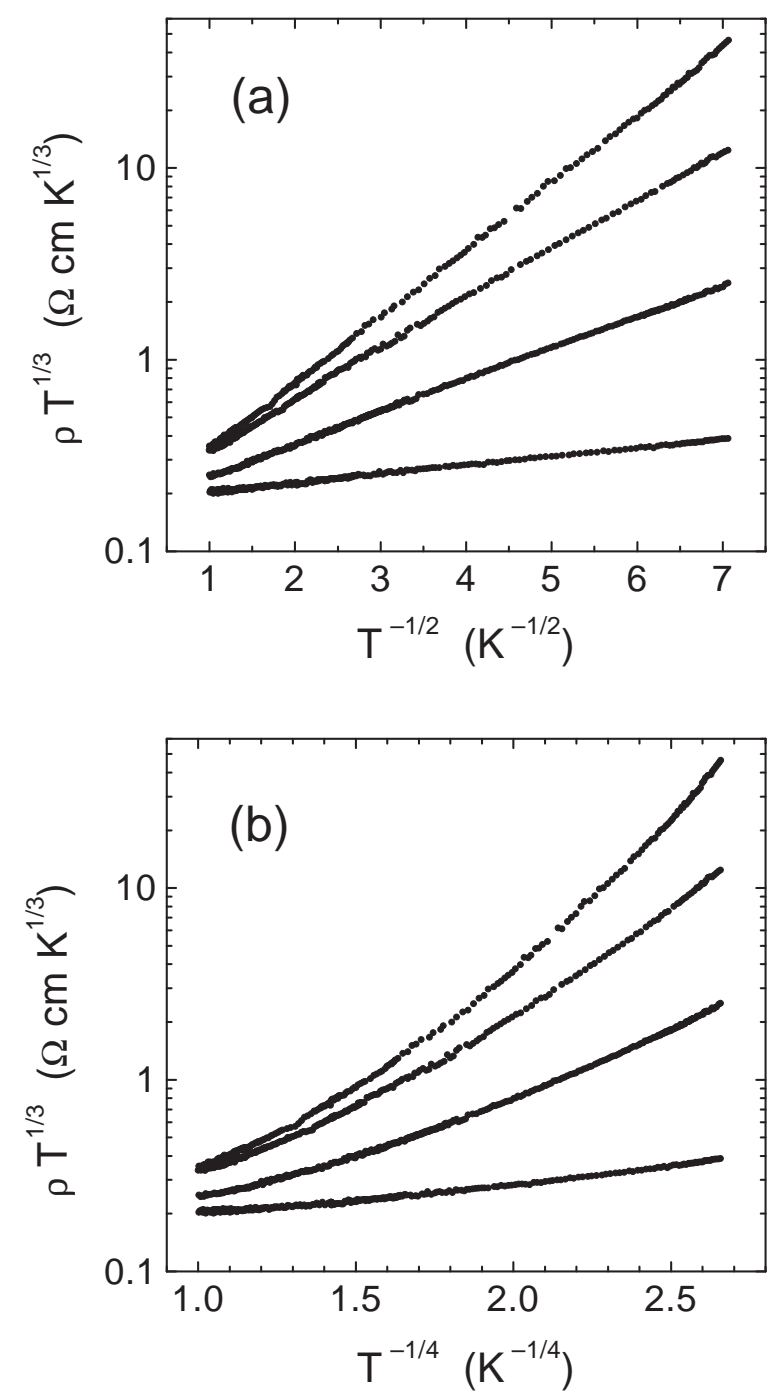

FIG. 1. Resistivity multiplied by $T^{1 / 3}$ vs (a) $T^{-1 / 2}$ and (b) $T^{-1 / 4}$ for ${ }^{70} \mathrm{Ge}$ Ga. From top to bottom in units of $10^{17} \mathrm{~cm}^{-3}$, the $\mathrm{Ga}$ concentrations are 1.848, 1.850, 1.853, and 1.856 .

Recently, we have shown that the temperature dependence of the conductivity of the same series of ${ }^{70} \mathrm{Ge}: \mathrm{Ga}$ samples within $\pm 0.3 \%$ of $N_{c}$ is proportional to $T^{1 / 3}$ at
$0.02-1 \mathrm{~K} .3$ Since both the $T^{1 / 3}$ dependence of the conductivity and the Efros-Shklovskii VRH are results of the electron-electron interaction in disordered systems, they can be expressed, in principle, in a unified form. Moreover, the electronic transport in barely metallic samples and that in barely insulating samples should be essentially the same at high temperatures so long as the inelastic scattering length and the thermal diffusion length are smaller than, or at most comparable to the correlation length or the localization length. So, the temperature dependence of conductivity at high temperatures should be the same on both sides of the transition. Such behavior is confirmed experimentally in the present system, 3 i.e., the conductivity of samples very close to $N_{c}$ shows a $T^{1 / 3}$ dependence at $T \approx 0.5 \mathrm{~K}$, irrespective of the phase (metal or insulator) to which they belong at $T=0$. Based on this consideration we fix $r=1 / 3$. Figure 11 shows $\rho T^{r}$ with $r=1 / 3$ for four samples $\left(N / N_{c}=0.993,0.994,0.996\right.$, and 0.998) as a function of (a) $T^{-1 / 2}$ and (b) $T^{-1 / 4}$. All the data points lie on straight lines with $p=1 / 2$ in Fig. 1(a) while they curve upward with $p=1 / 4$ in Fig. 1 (b). This dependence is maintained even when we change the values of $r$ between $1 / 2$ and $1 / 4$. Thus we conclude that the resistivity of all samples for $N$ up to $0.998 N_{c}$ is described by the VRH theory where the excitation occurs within the Coulomb gap, i.e., Eq. (11) with $p=1 / 2$.

Based on these findings, we evaluate $T_{0}$ in Eq. (1) with $p=1 / 2$ and $r=1 / 3$, and show it as a function of $1-N / N_{c}$ in Fig. 2. The vertical and horizontal error bars have been estimated based on the values of $T_{0}$ obtained with $r=1 / 2$ and $r=1 / 4$, and the values of $1-N /\left(1.858 \times 10^{17} \mathrm{~cm}^{-3}\right)$ and $1-N /\left(1.861 \times 10^{17} \mathrm{~cm}^{-3}\right)$, where $1.858 \times 10^{17} \mathrm{~cm}^{-3}$ is the highest concentration in the insulating phase and $1.861 \times 10^{17} \mathrm{~cm}^{-3}$ is the lowest in the metallic phase, respectively.

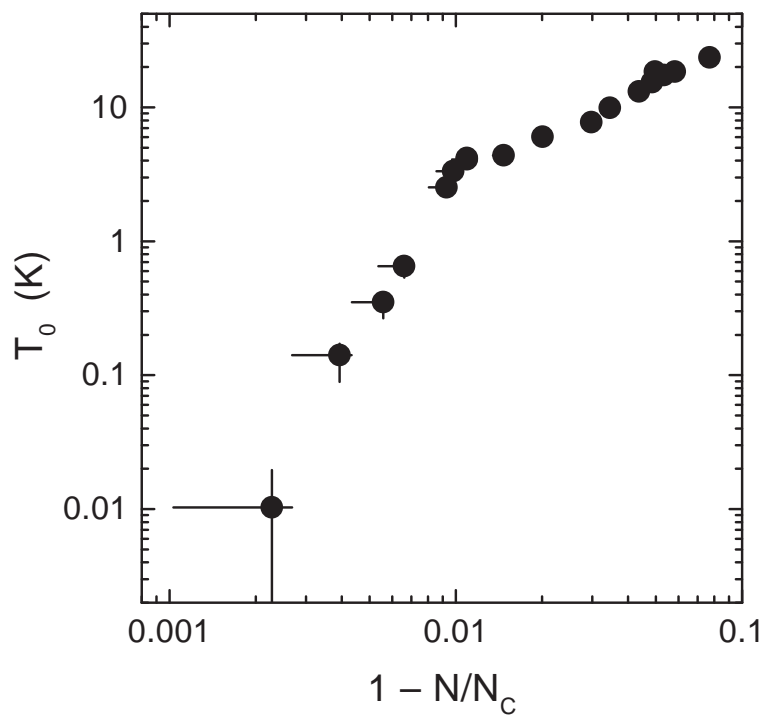

FIG. 2. $T_{0}$ determined by $\rho(T) \propto T^{-1 / 3} \exp \left[\left(T_{0} / T\right)^{1 / 2}\right]$ as a function of the dimensionless concentration $1-N / N_{c}$. 
According to theory, 目 $T_{0}$ in Eq. (1) is given by

$$
k_{B} T_{0} \approx 2.8 e^{2} / 4 \pi \epsilon_{0} \epsilon(N) \xi(N)
$$

in SI units, where $\epsilon(N)$ is the dielectric constant. Here, we should note that the condition $T<T_{0}$ is needed for the theory to be valid, i.e., $T_{0}$ has to be evaluated only from the data obtained at temperatures low enough to satisfy the condition. This requirement is fulfilled in Fig. 2 for all the samples except for the one with $N=0.998 N_{c}$. Concerning this latter sample, we will include it for the determination of $\xi$ and $\chi_{\text {imp }}$ (Fig. 5) but not for the calculation of the critical exponents.

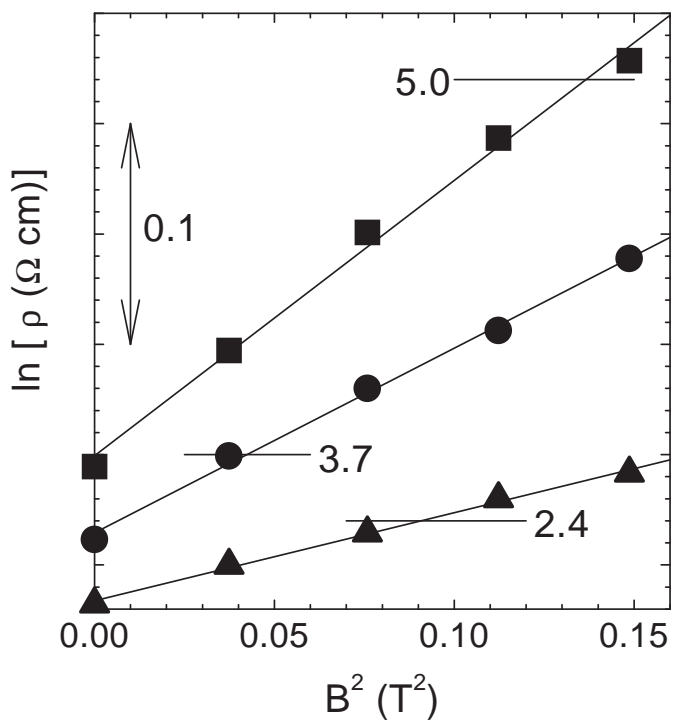

FIG. 3. Logarithm of resistivity vs $B^{2}$ at constant temperatures for the sample having $N=1.840 \times 10^{17} \mathrm{~cm}^{-3}$. From top to bottom the temperatures are $0.095 \mathrm{~K}, 0.135 \mathrm{~K}$, and $0.215 \mathrm{~K}$, respectively. The solid lines represent the best fits.

Our next step is to separate $T_{0}$ into $\epsilon$ and $\xi$. For $\xi / \lambda \ll 1$, the magnetoresistance is expressed as

$$
\ln [\rho(B, T) / \rho(0, T)] \approx 0.0015(\xi / \lambda)^{4}\left(T_{0} / T\right)^{3 / 2},
$$

where $\lambda \equiv \sqrt{\hbar / e B}$ is the magnetic length in SI units. According to Eq. (3), the magnetic-field variation of $\ln \rho$ at $T=$ const. is proportional to $B^{2}$, i.e., $\ln \rho(B, T)=$ $\ln \rho(0, T)+C(T) B^{2}$, and the slope $C(T)$ is proportional to $T^{-3 / 2}$. In order to demonstrate that these relations hold for our samples, we show for the $N=0.989 N_{c}$ sample $\ln \rho(B, T)$ vs $B^{2}$ in Fig. 3 and $C(T)$ determined by least-square fitting of $\partial \ln \rho / \partial B^{2}$ vs $T^{-3 / 2}$ in Fig. 1 . Since Eq. (3) is equivalent to

$$
\gamma \equiv C(T) / T^{-3 / 2} \approx 0.0015(e / \hbar)^{2} \xi^{4} T_{0}^{3 / 2},
$$

$\xi$ is given by

$$
\xi \approx 5.1(\hbar / e)^{1 / 2} \gamma^{1 / 4} T_{0}^{-3 / 8} .
$$

In this way we have determined $\gamma$ for nine samples. The inset of Fig. 5 shows $\gamma$ as a function of $T_{0}$. The value of $\gamma$ is almost independent of $T_{0}$, and if one assumes $\gamma \propto T_{0}^{\delta}$, one obtains a small value of $\delta=0.094 \pm 0.005$ from leastsquare fitting. Figure 5 shows $\xi$ and $\chi_{\mathrm{imp}}=\epsilon-\epsilon_{h}$ determined from Eqs. (2) and (5). Here, $\epsilon_{h}$ is the dielectric constant of the host Ge, and hence, $\chi_{\mathrm{imp}}$ is the dielectric susceptibility of the Ga acceptors. We should note that both $\xi$ and $\chi_{\mathrm{imp}}$ are sufficiently larger than the Bohr radius ( $8 \mathrm{~nm}$ for $\mathrm{Ge}$ ) and $\epsilon_{h}=15.4$ (Ref. 12), respectively. According to the theories of the MIT, both $\xi$ and $\chi_{\text {imp }}$ diverge at $N_{c}$ as $\xi(N) \propto\left(1-N / N_{c}\right)^{-\nu}$ and $\chi_{\text {imp }}(N) \propto\left(N_{c} / N-1\right)^{-\zeta}$, respectively. We find, however, both $\xi$ and $\chi_{\text {imp }}$ do not show such simple dependencies on $N$ in the range shown in Fig. 5 , and that there is a sharp change of both dependencies at $N \approx 0.99 N_{c}$. On both sides of the change in slope, the concentration dependence of $\xi$ and $\chi_{\text {imp }}$ are expressed well by the scaling formula as shown in Fig. 5 . Theoretically, the quantities should show the critical behavior when $N$ is very close to $N_{c}$. So $\nu=1.2 \pm 0.3$ and $\zeta=2.3 \pm 0.6$ may be concluded from the data in $0.99<N / N_{c}$. However, the other region $\left(0.9<N / N_{c}<0.99\right)$, where we obtain $\nu=0.33 \pm 0.03$ and $\zeta=0.62 \pm 0.05$, is also very close to $N_{c}$ in a conventional experimental sense.

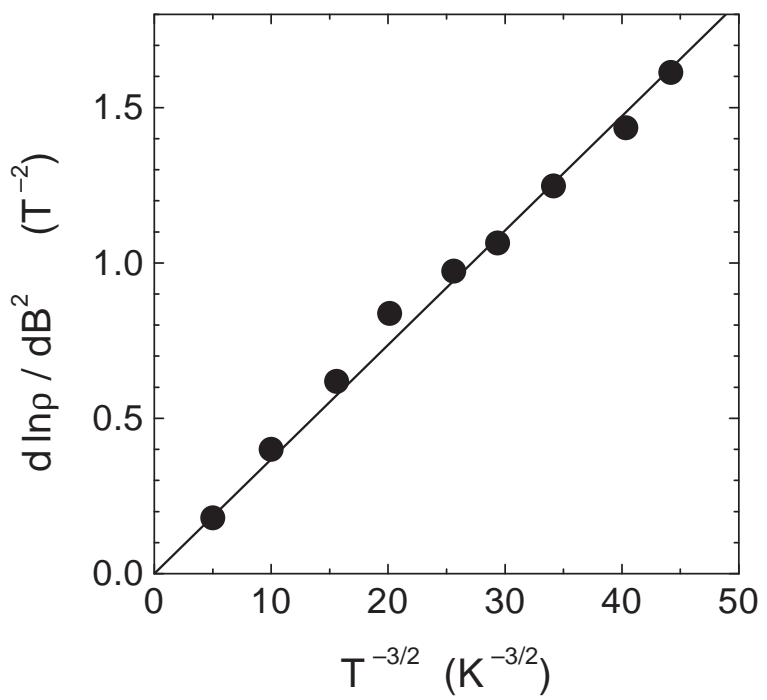

FIG. 4. Slope $d \ln \rho / d B^{2}$ vs $T^{-3 / 2}$ for the sample having $N=1.840 \times 10^{17} \mathrm{~cm}^{-3}$. The solid line represents the best fit.

As a possible origin for the change in slope, we refer to the effect of compensation. Although our samples are nominally uncompensated, doping compensation of less than $0.1 \%$ may present due to residual isotopes that become $n$-type impurities after NTD. In addition to the doping compensation, the effect known as "self compensation" may play an important role near $N_{c} .18$ It is empirically known that the doping compensation affects the value of the critical exponents. Rentzsch et al. studied VRH conduction of $n$-type Ge in the concentration range of $0.2<N / N_{c}<0.91$, and showed that $T_{0}$ vanishes as $T_{0} \propto\left(1-N / N_{c}\right)^{\alpha}$ with $\alpha \approx 3$ for $K=38 \%$ 
and $54 \%$, where $K$ is the compensation ratio 19 Since $\alpha \approx \nu+\zeta\left[\mathrm{Eq}\right.$. (2)], we find for our ${ }^{70} \mathrm{Ge}: \mathrm{Ga}$ samples $\alpha=3.5 \pm 0.8$ for $0.99<N / N_{c}<1$ and $\alpha=0.95 \pm 0.08$ for $0.9<N / N_{c}<0.99$. Interestingly, $\alpha=3.5 \pm 0.8$ agrees with $\alpha \approx 3$ found for compensated samples. Moreover, we have recently proposed the possibility that the conductivity critical exponent $\mu \approx 1$ in the same ${ }^{70} \mathrm{Ge}: \mathrm{Ga}$ only within the very vicinity of $N_{c}$ (up to about $+0.1 \%$ of $N_{c}$ ).6 An exponent of $\mu=0.50 \pm 0.04$, on the other hand, holds for a wide region of $N$ up to $1.4 N_{c}$. 3 Again, $\mu \approx 1$ near $N_{c}$ may be viewed as the effect of compensation. Therefore, it may be possible that the region of $N$ around $N_{c}$ where $\nu \approx 1$ and $\mu \approx 1$ changes its width as a function of the doping compensation. In the limit of zero compensation, the part which is characterized by $\nu \approx 1$ and $\mu \approx 1$ vanishes, i.e., we propose $\nu=0.33 \pm 0.03, \zeta=0.62 \pm 0.05$, and $\mu=0.50 \pm 0.04$ for truly uncompensated systems and that Wegner's scaling law of $\nu=\mu$ is not satisfied. In compensated systems, on the other hand, Wegner's law may hold as it does in the very vicinity of $N_{c}$. The experiment on compensated $\mathrm{Al}_{x} \mathrm{Ga}_{1-x} \mathrm{As}: \mathrm{Si}$ that showed $\zeta \approx 2$ and $\mu \approx 1$ (Ref. 14) is also consistent with the law. However, our preceding discussion needs to be proven in the future by experiments in samples with precisely and systematically controlled compensation ratios.

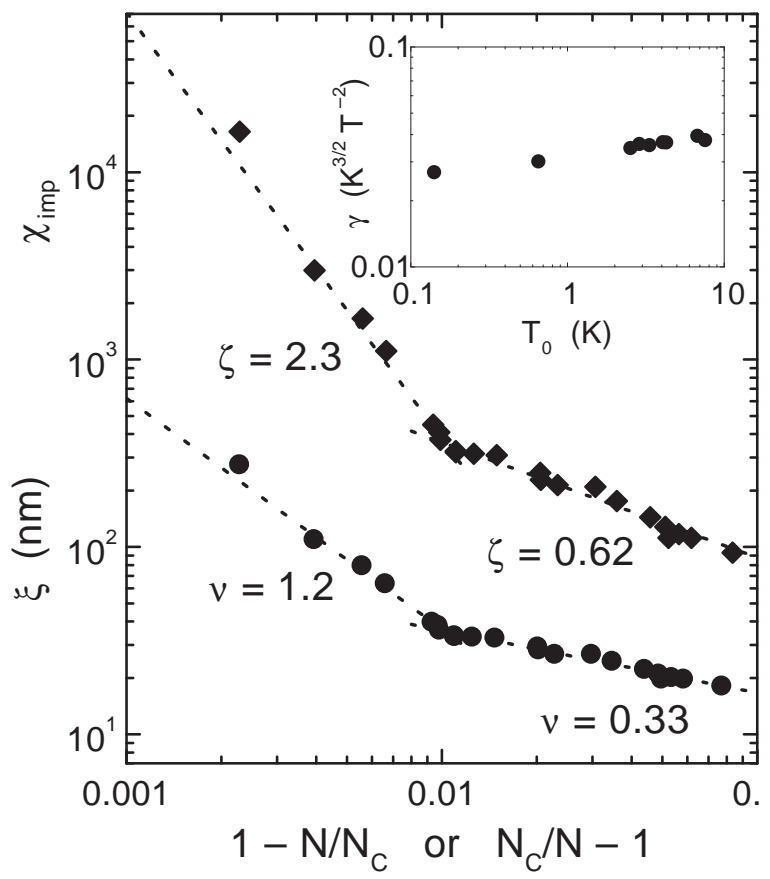

FIG. 5. Localization length $\xi$ vs $1-N / N_{c}$ (lower data set) and the dielectric susceptibility $\chi_{\mathrm{imp}}$ arising from the impurities vs $N_{c} / N-1$ (upper data set). The inset shows the coefficient $\gamma$ defined by Eq. (4) as a function of $T_{0}$.

In summary, we have determined directly the localization length and the dielectric susceptibility arising from the impurities in nominally uncompensated NTD ${ }^{70} \mathrm{Ge}$ Ga samples near the critical point for the MIT.
While the relation $2 \nu \approx \zeta$ predicted by scaling theory 10,11 holds for $0.9<N / N_{c}<1$, the critical exponents for localization length and impurity susceptibility change at $N / N_{c} \approx 0.99$. The small amount of doping compensation that is unavoidably present in our samples may be responsible for such a change in the exponents.

We are thankful to T. Ohtsuki, B. I. Shklovskii, and M. P. Sarachik for valuable comments, and V. I. Ozhogin for the supply of the Ge isotope. Most of the lowtemperature measurements were carried out at the Cryogenic Center, the University of Tokyo. M. W. would like to thank the Japan Society for the Promotion of Science (JSPS) for financial support. The work at Keio was supported by a Grant-in-Aid for Scientific Research from the Ministry of Education, Science, Sports, and Culture, Japan. The work at Berkeley was supported by the Director, Office of Energy Research, Office of Basic Energy Science, Materials Sciences Division of the U. S. Department of Energy under Contract No. DE-AC03-76SF00098 and U. S. NSF Grant No. DMR-97 32707.

${ }^{1}$ P. A. Lee and T. V. Ramakrishnan, Rev. Mod. Phys. 57, 287 (1985); D. Belitz and T. R. Kirkpatrick, ibid. 66, 261 (1994).

${ }^{2}$ K. M. Itoh et al., Phys. Rev. Lett 77, 4058 (1996).

${ }^{3}$ M. Watanabe et al., Phys. Rev. B 58, 9851 (1998).

${ }^{4}$ S. Bogdanovich, M. P. Sarachik, and R. N. Bhatt, Phys. Rev. Lett. 82, 137 (1999).

${ }^{5}$ S. Waffenschmidt, C. Pfleiderer, and H. v. Löhneysen, Phys. Rev. Lett. 83, 3005 (1999).

${ }^{6}$ K. M. Itoh et al., Ann. Phys. (Leipzig) 8, 631 (1999).

${ }^{7}$ F. J. Wegner, Z. Phys. B 25, 327 (1976).

${ }^{8}$ B. I. Shklovskii and A. L. Efros, Electronic Properties of Doped Semiconductors (Springer-Verlag, Berlin, 1984).

${ }^{9}$ A. N. Ionov, I. S. Shlimak, and M. N. Matveev, Solid State Commun. 47, 763 (1983).

${ }^{10}$ A. Kawabata, J. Phys. Soc. Jpn. 53, 318 (1984).

${ }^{11} \mathrm{Y}$. Imry, Y. Gefen, and D. J. Bergman, in Anderson Localization, Proceedings of the Fourth Taniguchi International Symposium, Sanda-Shi, Japan, 1981, edited by Y. Nagaoka and H. Fukuyama (Springer-Verlag, Heidelberg, 1982), p. 15.

12 T. G. Castner, Philos. Mag. B 42, 873 (1980).

${ }^{13}$ H. F. Hess et al., Phys. Rev. B 25, 5578 (1982).

${ }^{14} \mathrm{~S}$. Katsumoto, in Localization and Confinement of Electrons in Semiconductors, edited by F. Kuchar, H. Heinrich, and G. Bauer (Springer-Verlag, Berlin, 1990), p. 117.

${ }^{15}$ N. F. Mott, Metal-Insulator Transitions, 2nd ed. (Taylor \& Francis, London, 1990).

${ }^{16}$ B. I. Shklovskii (private communication).

17 See, for example, Sec. 4 in F. R. Allen and C. J. Adkins, Philos. Mag. 26, 1027 (1972).

${ }^{18}$ R. N. Bhatt and T. M. Rice, Philos. Mag. B, 42, 859 (1980).

${ }^{19}$ R. Rentzsch et al., Phys. Status Solidi B 205, 269 (1998). 